\title{
Caspase-3 suppresses diethylnitrosamine- induced hepatocyte death, compensatory proliferation and hepatocarcinogenesis through inhibiting p38 activation
}

\author{
Na Shang ${ }^{1}$, Thomas Bank ${ }^{1}$, Xianzhong Ding ${ }^{2}$, Peter Breslin ${ }^{1,3}$, Jun Li ${ }^{4}$, Baomin Shi ${ }^{5}$ and Wei Qiu ${ }^{1,5}$
}

\begin{abstract}
It is critical to understand the molecular mechanisms of hepatocarcinogenesis in order to prevent or treat hepatocellular carcinoma (HCC). The development of HCC is commonly associated with hepatocyte death and compensatory proliferation. However, the role of Caspase-3, a key apoptotic executor, in hepatocarcinogenesis is unknown. In this study, we used Caspase-3-deficient mice to examine the role of Caspase-3 in hepatocarcinogenesis in a chemical (diethylnitrosamine, DEN)-induced HCC model. We found that Caspase-3 deficiency significantly increased DEN-induced HCC. Unexpectedly, Caspase-3 deficiency increased apoptosis induced by DEN and the subsequent compensatory proliferation. Intriguingly, we discovered that Caspase-3 deficiency increased the activation of p38 with and without DEN treatment. Moreover, we demonstrated that TNFa and IL1a stimulated increased activation of p38 in Caspase-3 KO hepatocytes compared with wild-type hepatocytes. Finally, we found that inhibition of p38 by SB202190 abrogated enhanced hepatocyte death, compensatory proliferation and HCC induced by DEN in Caspase-3-deficient mice. Overall, our data suggest that Caspase-3 inhibits chemical-induced hepatocarcinogenesis by suppressing p38 activation and hepatocyte death.
\end{abstract}

\section{Introduction}

Hepatocellular carcinoma (HCC) is the second leading cause of cancer deaths worldwide ${ }^{1}$. The overall survival of patients with HCC is $<12 \%$, and most patients with HCC have limited treatment options ${ }^{2}$. Currently, the most effective targeted therapeutic agent for advanced HCC, sorafenib, only increases survival in patients with advanced HCC from 7.9 months to 10.7 months $^{3}$. There is an urgent need to develop more effective therapeutic strategies and agents to treat HCC. To achieve this goal,

\footnotetext{
Correspondence: Baomin Shi (baomingsph@163.com) or

Wei Qiu (wqiu@luc.edu)

${ }^{1}$ Department of Surgery and Oncology Institute, Loyola University Chicago

Stritch School of Medicine, 2160 South 1st Avenue, Maywood, IL 60153, USA

${ }^{2}$ Department of Pathology, Loyola University Chicago Stritch School of

Medicine, 2160 South 1st Avenue, Maywood, IL 60153, USA

Full list of author information is available at the end of the article.

Edited by E. Baehrecke
}

the molecular signaling pathways that drive or mediate the development of HCC must be better understood.

HCC is considered a chronic inflammation-related disorder, the development of which is commonly associated with hepatocyte death and compensatory proliferation ${ }^{1,4-10}$. Several classes of chemicals promote HCC in rodents, including diethylnitrosamine (DEN), which has been extensively studied. In the DEN-induced HCC model, DEN stimulates DNA damage and causes reactive oxygen species to accumulate; it also induces hepatocyte death through the c-Jun N-terminal kinase (JNK) pathway ${ }^{11-14}$. The dying cells release interleukin1 alpha (IL1 $\alpha)$, which activates Kupffer cells ${ }^{6,15}$. Activated Kupffer cells produce cytokines and growth factors such as interleukin-6 (IL-6) and tumor necrosis factor alpha $(\mathrm{TNF} \alpha)^{5,16-18}$, which promote expansion of hepatocytes with DNA mutations thus enhancing HCC 

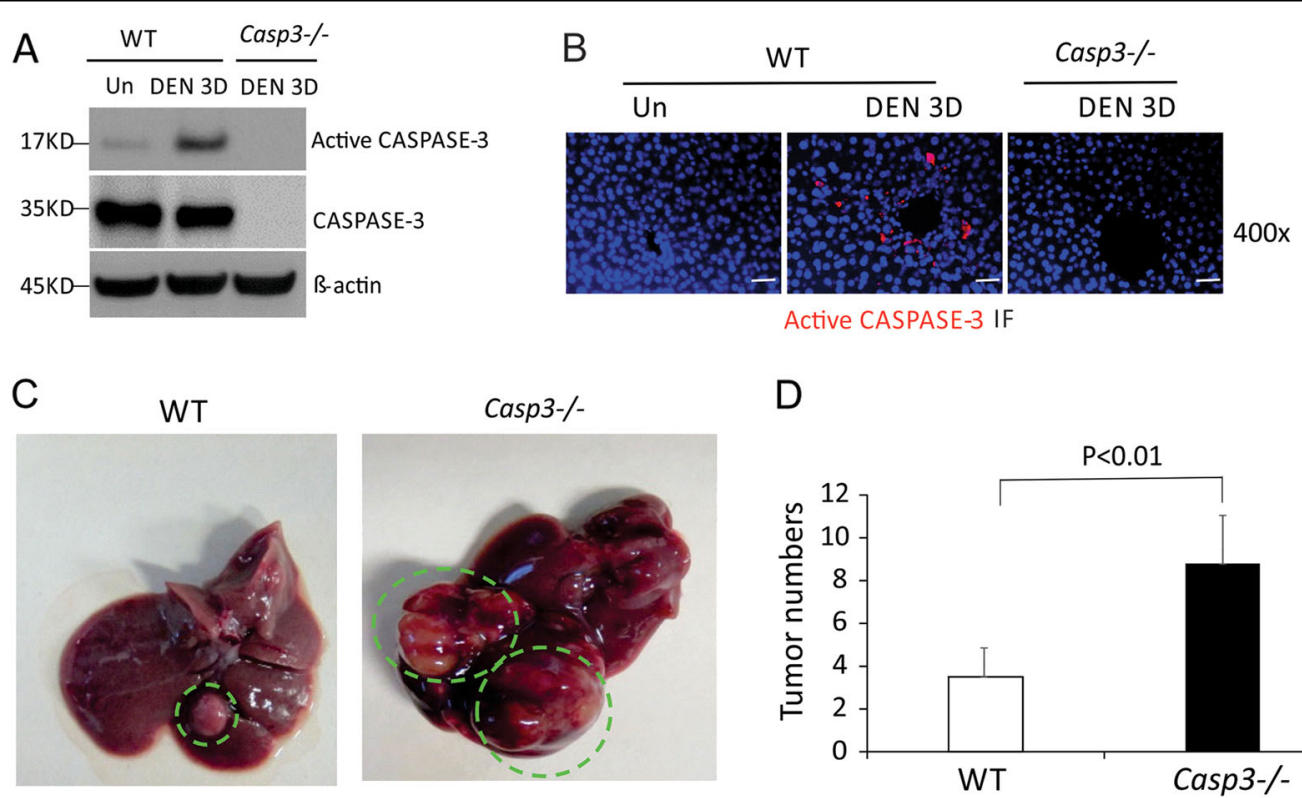

$\mathrm{D}$

DEN 9 months

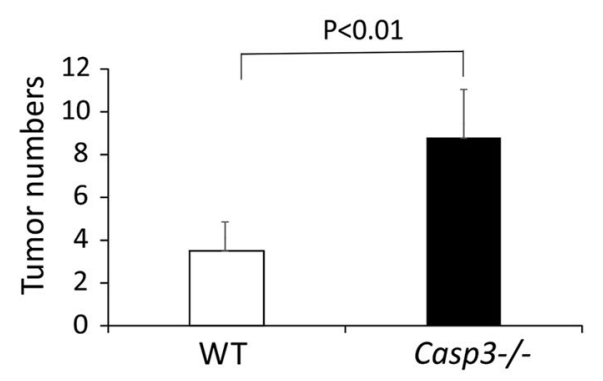

E

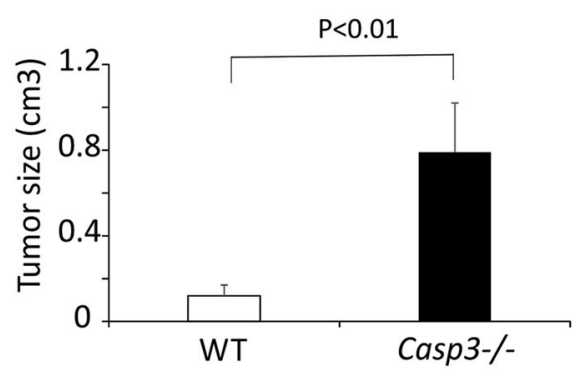

\section{$\mathrm{F}$}

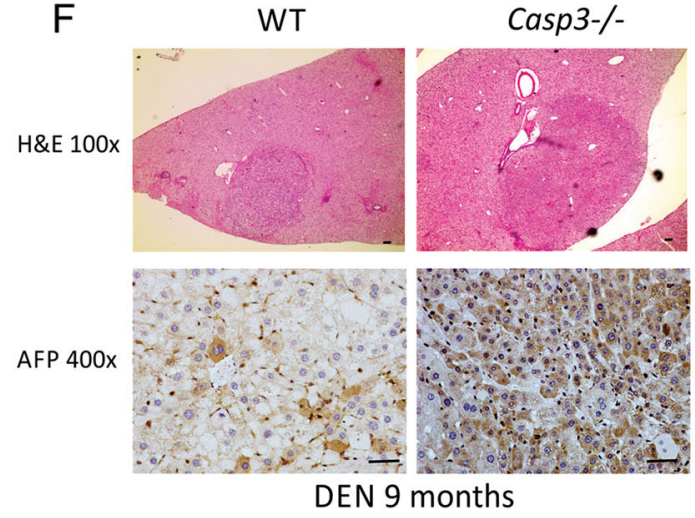

Fig. 1 Caspase-3 deficiency promoted DEN-induced liver cancer. a CASPASE-3, active CASPASE-3 and $\beta$-actin protein expression in the livers of WT mice 3 days following injection with either saline (Un) or $100 \mathrm{mg} / \mathrm{kg}$ of DEN was analyzed by western blotting. Values are means $\pm S D, n=3$ mice in each group. $\mathbf{b}$ Active CASPAPS-3 protein (red) in the livers of WT and Caspase-3 KO mice following indicated treatment was detected by IF with nuclei counterstained with DAPI (magnification, $\times 400$ ). c Photographs of livers of WT and Caspase-3 KO mice 9 months after DEN injection. d Quantification of liver tumor numbers $(n=6)$. e Quantification of liver tumor sizes $(n=6)$. $\mathbf{f}$ Histological analysis (H\&E staining) and staining of AFP of the livers of WT and Caspase-3 KO mice 9 months after DEN injection. Bars: $20 \mu \mathrm{M}$. Values in (d) and (e) are means \pm SDs

development ${ }^{5,16,17}$. We previously reported that deficiency of PUMA (p53 upregulated modulator of apoptosis) significantly decreased DEN-induced liver cancer by blocking acute apoptotic responses and the subsequent compensatory proliferation $^{19}$. These data provide evidence for the correlation of specific mediators of hepatocyte apoptosis and the development of HCC. Caspase-3 is a member of the cysteine-aspartic acid protease family (caspases) and plays a central role in the execution-phase of cellular apoptosis ${ }^{20}$. Given the strong association of apoptosis and compensatory proliferation in HCC development, and the specific apoptotic executive function of Caspase-3, we hypothesized that inhibition of Caspase-3 would suppress DNA damage-induced hepatocyte death, and thereby inhibit hepatic carcinogenesis.

Unexpectedly, we found that Caspase-3 deficiency significantly promoted DEN-induced HCC development. Intriguingly, Caspase-3 deficiency increased hepatocyte death and the subsequent compensatory proliferation induced by DEN. In addition, we discovered that Caspase3 deficiency increased the activation of p38 MAP kinase. Moreover, we demonstrated that deletion of Caspase- 3 increased p38 activation by IL $1 \alpha$ and TNF $\alpha$ in primary mouse hepatocytes. Furthermore, we found that inhibition of p38 abrogated enhanced hepatocyte death, the compensatory proliferation and HCCs induced by DEN in 


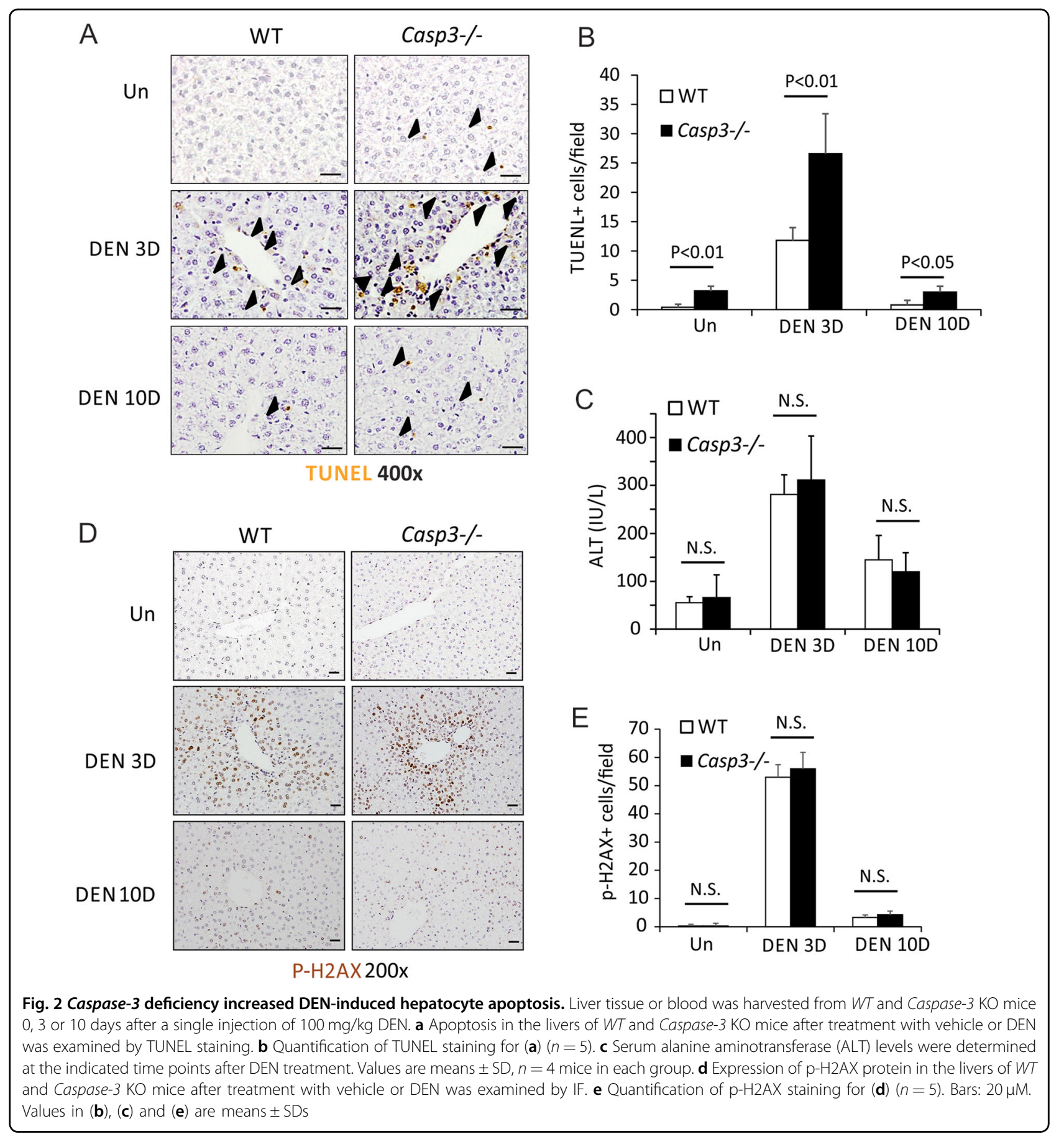

Caspase-3-deficient mice. Overall, our data suggest that Caspase-3 inhibits chemical-induced hepatocarcinogenesis by suppressing p38 activation and hepatocyte death.

\section{Results}

DEN induced Caspase-3 cleavage in mouse livers

To determine the role of Caspase- 3 in hepatocarcinogenesis, we used the DEN-induced HCC model because Caspase- 3 is cleaved and activated in response to DNA damage or non-genotoxic stimuli ${ }^{21}$ and DEN is known to induce DNA damage ${ }^{22}$. We analyzed the activation of Caspase-3 in the livers of WT mice after DEN treatment. Cleaved Caspase-3 but not total Caspase-3 was significantly elevated by day 3 after treatment compared with untreated mice (Fig. 1a). Immunostaining indicated that cleaved Caspase-3 protein was selectively induced in hepatocytes around the centrilobular regions $24 \mathrm{~h}$ after DEN treatment, whereas the basal level was undetectable (Fig. 1b). 
Caspase-3 deficiency promoted DEN-induced liver cancer

A single injection of DEN to 15-day-old male mice results in efficient $\mathrm{HCC}$ induction ${ }^{19}$. To determine a potential role for Caspase- 3 in hepatocarcinogenesis, we compared tumor incidence and size in WT and Caspase-3 knockout (Casp3 KO) littermates 9 months after DEN treatment. All of the mice developed tumors by 9 months of age (Fig. 1c). Interestingly, tumor incidence in Casp3 $\mathrm{KO}$ mice increased by about twofold compared with WT mice ( $8.8 \pm 2.2$ vs. $3.5 \pm 0.8)$ (Figs. 1 c, d). Tumor size was also significantly increased in Casp3 $\mathrm{KO}$ mice compared with WT mice (Figs. 1e, f). In addition, immunohistochemical (IHC) staining signals for alpha-fetoprotein (AFP), a common HCC marker, were significantly lower in the livers of WT mice compared with those of Casp3 KO mice (Fig. 1f). These results indicate that Casp3 deficiency promotes DEN-induced hepatocarcinogenesis.

\section{Caspase-3 deficiency increased DEN-induced hepatocyte death}

Caspase-3 is a critical apoptotic executor ${ }^{21}$. Promotion of HCC development by Casp 3 deficiency could be due to decreased apoptosis of tumor cells. To determine whether a deficiency of Casp 3 affects apoptosis in DEN-induced $\mathrm{HCC}$, we performed Terminal deoxynucleotidyl transferase dUTP nick end labeling (TUNEL) staining in liver tumors from WT and Casp3 KO mice treated with DEN. We did not find significant differences in apoptosis in tumors between these two groups of mice (Fig. S1A and $\mathrm{S} 1 \mathrm{~B})$. We also analyzed proliferation in the DEN-treated livers from $W T$ and Casp3 KO mice by Ki67 staining. The number of $\mathrm{Ki}^{+} 7^{+}$cells was comparable in Casp3-deficient and WT liver tumors (Fig. S1A and S1B). These results show that Casp3 deficiency affects neither apoptosis nor proliferation in DEN-induced HCC.

The development of DEN-induced HCC is associated with hepatocyte death and compensatory proliferation $^{1,4-10}$. DEN induces significant hepatocyte death in WT mice within 3 days (Figs. 2a, b), notably, in the centrilobular regions where Caspase- 3 is activated (Figs. 1b, 2a). Interestingly, there was dramatically enhanced hepatocyte death in Casp3 KO mice compared with WT mice after DEN treatment (Figs. 2a, b). Hepatocyte death decreased by 10 days after DEN treatment in both $W T$ and Casp3 KO mice, but was greater in Casp3 KO mice than in WT mice (Figs. 2a, b). DEN treatment increased serum levels of the liver enzyme alanine aminotransferase (ALT) by day 3, such increase being similar in both Casp3 $\mathrm{KO}$ and WT mice (Fig. 2c). As DEN can induce oxidative DNA damage in the liver ${ }^{5}$, we evaluated DNA double-strand breaks in livers by $\mathrm{p}$ H2AX staining. No difference in DNA damage was found between $W T$ and Casp3 $\mathrm{KO}$ mice 3 or 10 days after DEN treatment (Figs. 2d, e).
Caspase- 6 and Caspase- 7 may play similar functions as Caspase-3 in the execution of apoptosis ${ }^{23}$. To determine if deletion of Caspase-3 increases the activation of Caspase6 and Caspase- 7 after DEN treatment in mouse livers, we examined the expression of total and cleaved Caspase- 6 and Caspase-7 in DEN-treated mouse livers. We found a slight decrease in cleavage of Caspase- 6 or Caspase-7 in Casp3 KO mice compared with WT mice (Fig. S2), suggesting that increased DEN-induced hepatocyte apoptosis resulting from Caspase-3 deficiency is not the result of enhanced activation of Caspase- 6 or Caspase-7 in DENtreated mouse livers.

\section{Caspase-3 deficiency enhanced DEN-induced compensatory proliferation}

DEN-induced hepatocyte death is associated with compensatory proliferation and HCC development ${ }^{19}$. As expected, elevated proliferation was found in the livers of WT mice 3 and 10 days following DEN treatment as evidenced by Ki67 and PCNA staining (Figs. 3a-d). The proliferation centered on the centrilobular regions where apoptotic cells were detected (Figs. 2a, 3a). The degree of proliferation was significantly increased in Casp3 $\mathrm{KO}$ mice compared with WT mice (Figs. 3a-d). IL-6 and its downstream target Stat3 have been shown to play important roles in DEN-induced compensatory proliferation ${ }^{5,16-18}$. We therefore examined the expression of IL-6 mRNA and phosphorylation of Stat3 in WT and Casp3 KO mouse livers. We found that IL- 6 mRNA was increased in Caspase- $3 \mathrm{KO}$ mouse livers compared with WT mouse livers after DEN treatment both on day 3 and day 10 (Fig. S3). Phosphorylation of Stat3 was also increased in Caspase-3 KO mouse livers compared with WT mouse livers after DEN treatment on day 3 while the difference was diminished by day 10 (Fig. S3). These results showed that Caspase 3 deficiency promotes DENinduced compensatory proliferation in hepatocytes.

\section{Caspase-3 deficiency enhanced p38 activation in mouse livers}

Activation of JNK, nuclear factor-kB (NF-kB) and p38 by cytokines, including TNF $\alpha$ and IL $1 \alpha$, plays a critical role in hepatocyte death and compensatory proliferation after DEN treatment ${ }^{24,25}$. To determine whether deletion of Caspase-3 affects the activation of JNK, NF- $\mathrm{kB}$ and p38 in the liver after DEN treatment, we compared the phosphorylation of these three proteins in the livers of WT and Casp3 KO mice. There was no significant difference in the phosphorylation status of JNK in Casp3 KO mouse livers compared with WT mouse livers prior to or after DEN treatment (Fig. 4a). NF- $\mathrm{KB}$ was slightly increased by Caspase-3 deficiency in response to DEN treatment by 3 days but not after 10 days (Fig. 4a). Accordingly, phosphorylation of I-kappa-B-alpha (I $\mathrm{B} \mathrm{B} \alpha)$ 


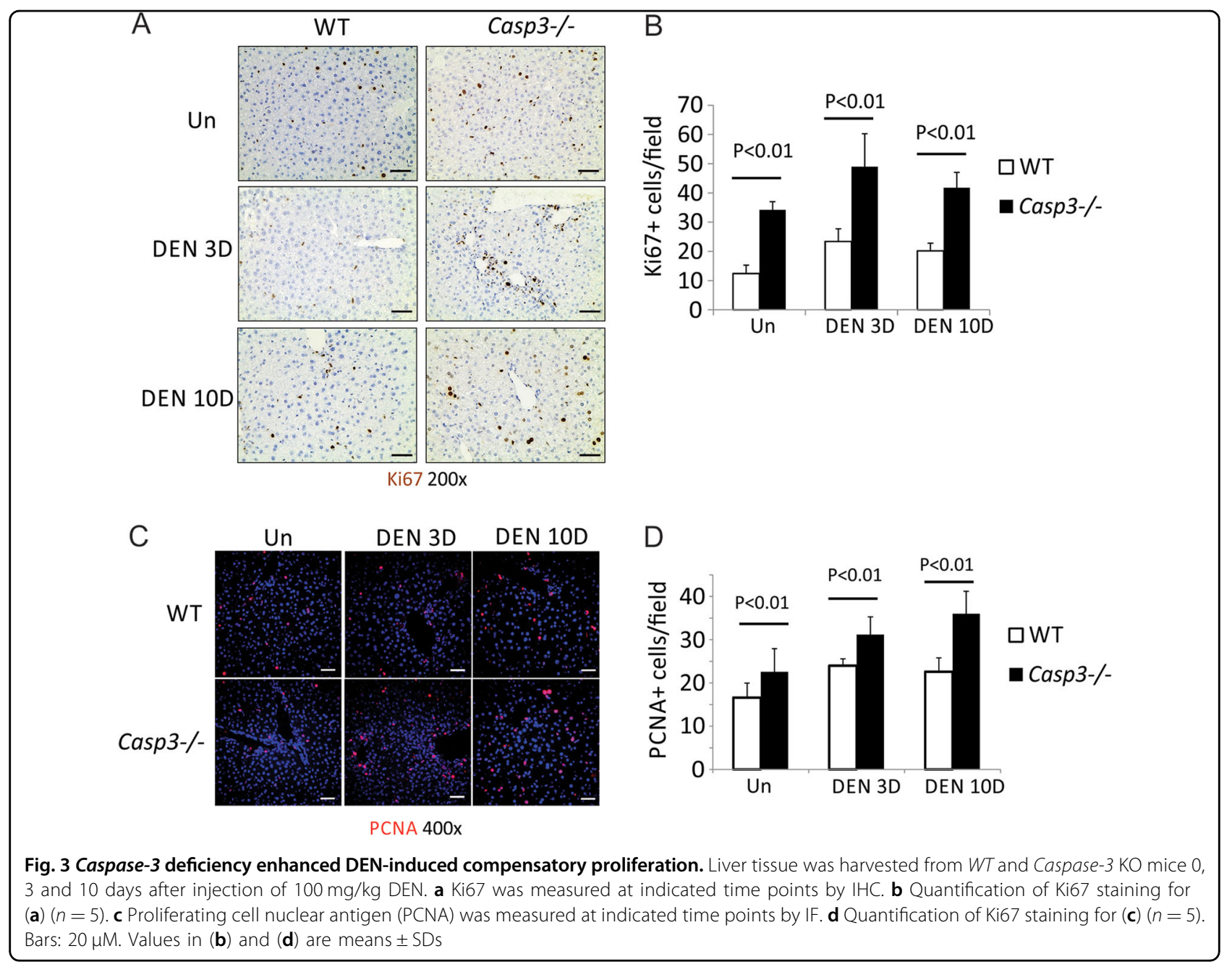

on Ser 32 and Ser 36, which results in release and nuclear translocation of active NF- $\mathrm{B}^{26}$, was also slightly increased by Caspase- 3 deficiency in response to DEN treatment on day 3 after treatment (Fig. S4A). We also examined levels of IкB $\alpha$, Vcam 1 and Cxcl10 mRNA, which have been reported to be direct target genes of NF$\mathrm{KB}^{27,28}$. We found that the expression of Vcam1 and Cxcl10 but not IKB $\alpha$ was increased by Caspase-3 deficiency in response to DEN treatment in mouse livers (Fig. $\mathrm{S} 4 \mathrm{~B})$. NF- $\mathrm{kB}$ has been shown to protect against DENinduced hepatocyte death and $\mathrm{HCC}^{5,24}$. Therefore, it is unlikely that an increase in NF- $\kappa B$ activation led to enhanced DEN-induced hepatocyte death, compensatory proliferation and HCC caused by Caspase-3 deficiency. Interestingly, higher p-p38 was observed in Casp3 KO mouse livers compared with WT mouse livers with or without DEN treatment (Fig. 4a), suggesting that deletion of Caspase-3 increases the activation of p38 in mouse livers. Consistently, phosphorylation of MK2, a downstream direct target of $\mathrm{p} 38$, was also increased in Casp3
KO mouse livers (Fig. S5A). MMK6 and MMK3 have been shown to phosphorylate and activate p38. We also found that phosphorylation of MAP kinase kinases $3 / 6$ (MKK3/6) was increased in Casp3 KO mouse livers (Fig. S5A).

\section{Caspase-3 deficiency did not affect TNFa nor IL1a expression in mouse livers after DEN treatment}

TNF $\alpha$ plays a critical role in hepatocyte compensatory proliferation and the activation of JNK, NF- $\mathrm{kB}$ and p38 after DEN treatment ${ }^{24,25}$. It is possible that Caspase-3 deficiency enhances p38 activation in mouse liver by increasing the expression of TNF $\alpha$ after DEN treatment. We therefore compared mRNA and protein levels of TNF $\alpha$ in the livers of WT and Casp3 KO mice. Expression of both TNF $\alpha$ mRNA and protein was increased when examined 3 days following DEN treatment in WT mice, whereas comparable expression of TNFa was observed in Casp3 KO mice (Fig. 4b and Fig. S5A). IL1 $\alpha$ has been shown to phosphorylate and activate p38 in liver 

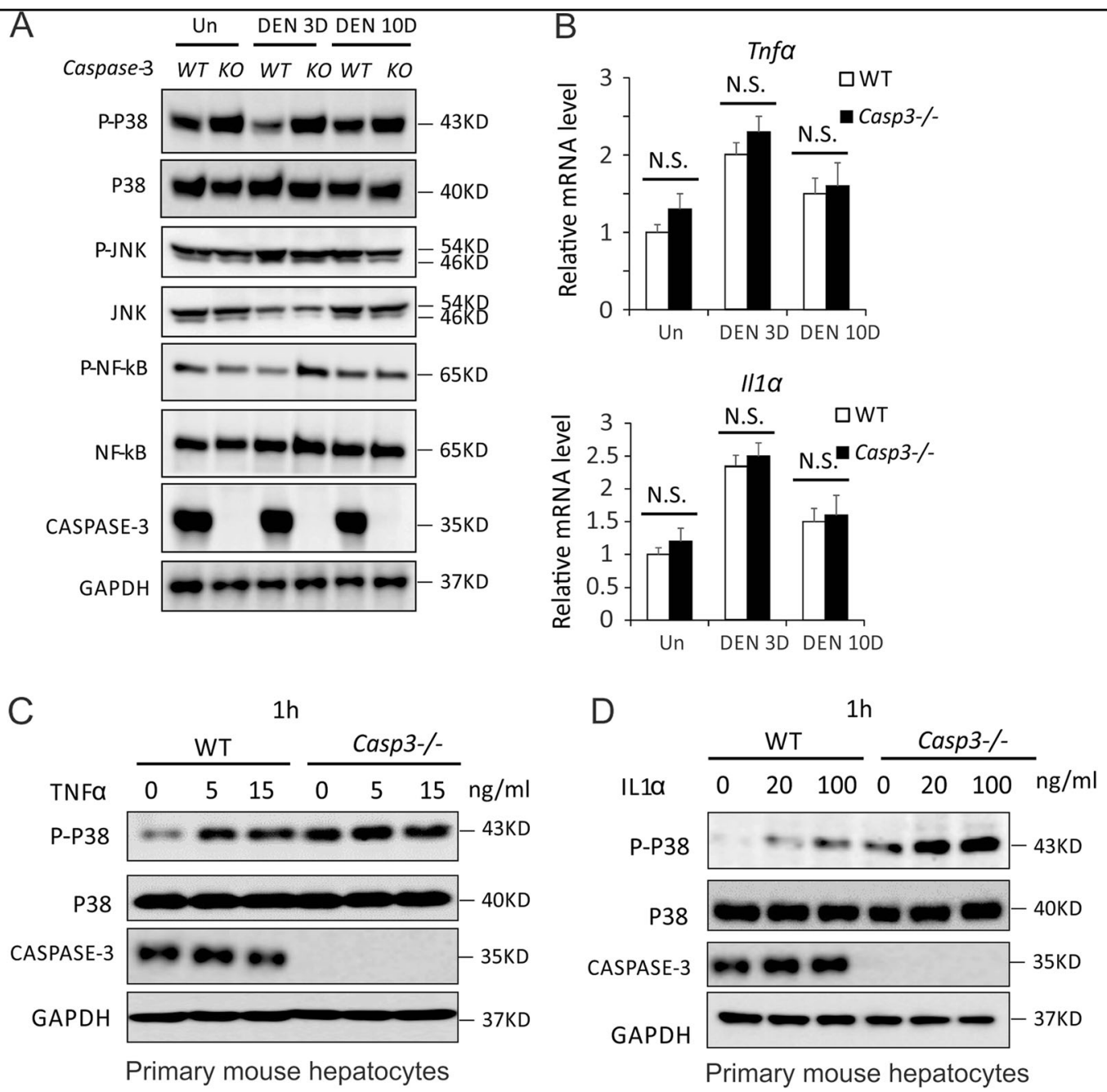

Fig. 4 Caspase-3 deficiency enhanced TNFa- or IL1a-induced p38 activation in mouse hepatocytes. a Expression of p-p38, p38, p-JNK, JNK, p-NF-KB, NF-KB, CASPASE-3 and GAPDH proteins in the livers of WT and Caspase-3 KO mice 0, 3 and 10 days following injection with either saline (Un) or $100 \mathrm{mg} / \mathrm{kg}$ of DEN was analyzed by western blotting. $\mathbf{b}$ mRNA expression of Tnfa and IL $1 \mathrm{a}$ in the livers of WT and Caspase-3 KO mice 0, 3 and 10 days following injection with either saline (Un) or $100 \mathrm{mg} / \mathrm{kg}$ of DEN was analyzed by western blotting. Values are means $\pm S D s, n=3$ mice in each group. c Expression of p-p38, p38, CASPASE-3 and GAPDH proteins in isolated hepatocytes from WT and Caspase-3 KO mice $1 \mathrm{~h}$ after treatment with 0,5 or $15 \mathrm{ng} / \mathrm{ml}$ TNFa was analyzed by western blotting. $\mathbf{d}$ Expression of p-p38, p38, CASPASE-3 and GAPDH proteins in isolated hepatocytes from WT and Caspase-3 KO mice $1 \mathrm{~h}$ after treatment with 0, 20 or $100 \mathrm{ng} / \mathrm{ml} \mathrm{IL} 1 \mathrm{a}$ was analyzed by western blotting. Values in (b) are means \pm SDs

cells $^{29}$. We therefore examined the expression of IL1 $\alpha$ and found no significant difference in IL1 $\alpha$ mRNA expression (Fig. 4b). These data indicate that Caspase-3 deficiency increases the activation of p38 but does not do so through enhanced expression of TNF $\alpha$ or IL1 $\alpha$ in mouse livers.

\section{Caspase-3 deficiency enhanced activation of p38} by in response to TNFa or IL1a in hepatocytes

As the expression of neither TNF $\alpha$ nor $I L 1 \alpha$ was affected in mouse livers by deletion of Caspase-3, we therefore examined whether Caspase-3 deficiency in hepatocytes enhances the phosphorylation of p38 in response to TNF $\alpha$ or IL $1 \alpha$. We isolated primary hepatocytes from WT and Casp $3 \mathrm{KO}$ mice and treated them with TNF $\alpha$ or IL1 $\alpha$. There was a significant enhancement of p38 phosphorylation in Casp3 KO hepatocytes compared with $W T$ hepatocytes prior to or after treatment with TNF $\alpha$ (Fig. 4c). Similarly, phosphorylation of p38 was increased in Casp3 $\mathrm{KO}$ hepatocytes compared with WT hepatocytes after $1 \mathrm{~h}$ of IL1 $\alpha$ treatment (Fig. 4d). Consistently, phosphorylation of MK2 and MKK3/6 was also increased in Casp3 KO hepatocytes compared with $W T$ hepatocytes after $1 \mathrm{~h}$ of treatment with IL1 $\alpha$ or TNF $\alpha$ (Fig. S5B and C). In general, these data demonstrate that Caspase-3 deficiency enhances 
the activation of $\mathrm{p} 38$ in hepatocytes in response to $T N F \alpha$ or $I L 1 \alpha$.

\section{Inhibition of p38 abrogated enhanced DEN-induced hepatocyte death, compensatory proliferation and HCC development induced by deletion of Caspase-3}

Emerging evidence indicates that activation of p38 promotes cell death. Activation of $\mathrm{p} 38$ by MKK6 has been shown to induce apoptosis in Huh7 and HepG2 cells $^{30}$. A p38 MAPK inhibitor markedly suppressed cell death induced by $\mathrm{TNF}^{31}$. Therefore, we reasoned that Caspase3 deficiency increased DEN-induced hepatocyte apoptosis through enhanced p38 activation. To test this hypothesis, we first examined whether the overexpression of p38 increases TNF $\alpha$-induced cell death in AML12 cells, a mouse hepatocyte cell line. We found that overexpression of p38 did increase TNF $\alpha$-induced cell death in these cells (Fig. S6). Further, we treated WT and Casp3 $\mathrm{KO}$ mice with a specific inhibitor of p38, SB202190, prior to and after DEN treatment. We found that SB202190 decreased the phosphorylation of p38 in both WT and Casp3 KO mice (Fig. 5a) and inhibited DEN-induced hepatocyte death (Figs. 5b, c). Consistently, SB202190 also decreased DEN-induced compensatory proliferation in Casp3 KO mice (Fig. 5d). Importantly, SB202910 inhibited DENinduced HCC development in Casp3 KO mice (Figs. 5e, f). These data suggest that deletion of Caspase-3 enhances DEN-induced hepatocyte death, compensatory proliferation and the development of HCC by increasing the activation of $\mathrm{p} 38$.

\section{Discussion}

Treatment options for HCC are limited ${ }^{2}$. Currently, the most effective targeted therapeutic agent for advanced HCC, sorafenib, an inhibitor of several protein tyrosine kinases (including vascular endothelial growth factor receptor and platelet-deried growth factor receptor) and Raf kinases, increases survival in patients with advanced HCC by only 3 months ${ }^{3}$. More effective therapeutic strategies and agents to treat HCC are clearly needed. Therefore, it is necessary to elucidate the molecular signaling pathways that drive or mediate the development of HCC. Hepatocyte apoptosis has been shown to play an important role in HCC development. About $3.5 \%$ of HCC samples from The Cancer Genome Atlas (TCGA) liver HCC data sets show deletion of the Caspase-3 gene, which is correlated with reduced Caspase-3 mRNA expression (Fig. S7). A recent study indicated that low expression of Caspase-3 is correlated with poor prognosis in HCC patients ${ }^{32}$, suggesting that Caspase-3 might be involved in HCC pathogenesis. However, the role of Caspase-3 in HCC development in vivo has not been reported. In this study, we found that deletion of Caspase3 promotes DEN-induced hepatocyte death, compensatory proliferation and carcinogenesis, indicating that Caspase-3 plays an inhibitory role in hepatocarcinogenesis. Therefore, it is likely that Caspase-3 deletion or downregulation in human HCC samples promotes HCC development.

Caspase-3 is a central executor of apoptosis. Therefore, it was surprising to us that Caspase- 3 deletion increases apoptosis induced by DEN in mouse livers. Caspase- 6 and Caspase-7, the other two apoptotic executors, have been shown to compensate the functions of Caspase- 3 in some cell contexts ${ }^{33}$. However, we observed no compensatory activation of Caspase-6 or Caspase-7 in DEN-treated WT or Caspase-3 KO livers. Instead, we found that loss of Caspase- 3 increases the activation of $\mathrm{p} 38$ in response to DEN, TNF $\alpha$ or IL1 $\alpha$ in hepatocytes. It is notable that p38 hyperactivation in Casp3 $\mathrm{KO}$ mice is largely constitutive (Fig. 4a), although treatment with IL1 $\alpha$ or TNF $\alpha$ promotes a further increase in phosphorylation (Fig. S5B). It is possible that Caspase-3 deletion increases p38 activation in response to the basal level of TNF $\alpha$ or IL1 $\alpha$ in mouse liver. Activation of p38 by MKK6 has been shown to induce apoptosis in $\mathrm{HCC}$ cells ${ }^{30}$. p38 also mediates cell death induced by TNF $\alpha$ in cancer cells ${ }^{31}$. We also found that overexpression of $\mathrm{p} 38$ enhanced TNF $\alpha$-induced cell death in mouse liver cells (Fig. S6). In addition, we demonstrated that a p38 inhibitor abrogates the enhanced DEN-induced hepatocyte death induced by deletion of Caspase-3. Overall, our data suggest that Casp3 KO hepatocytes are more sensitive to TNF $\alpha$, which is likely due to $\mathrm{p} 38$ hyperactiation (Fig. S6). Therefore, deletion of Caspase-3 enhances DEN-induced hepatocyte death, compensatory proliferation and hepatocarcinogenesis by increasing the activation of p38 (Fig. 6). To our knowledge, this is the first report of inhibition of p38 activation by Casp-3. It is unknown whether this mechanism is a general one that will be found all in cell types or whether it is in cell context specific. It will be interesting for us to test this question in all our future studies.

Increased activation of p38 caused by the deletion of Caspase-3 may be due to the result of multiple actions. MKKs 3 and 6 and have been identified as common activators of p38 in the liver ${ }^{34}$. Indeed we found that phosphorylation of MKK6/3 is increased in Caspase-3deficient hepatocytes (Fig. S4), suggesting that Caspase-3 deletion increases the activation of p38 through enhancing MKK6/3 activation in hepatocytes. Several MAP kinase kinase kinases have been shown to trigger MKK6/ 3 activation; they include apoptosis signal-regulating kinase 1 (ASK1), dual-leucine zipper-bearing kinase 1 (DLK1), thousand-and-one amino acid (TAO) 1 and 2, tumor progression loci 2 (TPL2), mixed lineage kinase 3 (MLK3), MEK kinase 3 (MEKK3), MEKK4, and leucine zipper and sterile- $\alpha$ motif kinase 1 (ZAK1) ${ }^{29}$. Over three hundred targets of Caspase- 3 have been identified ${ }^{35}$. It is 


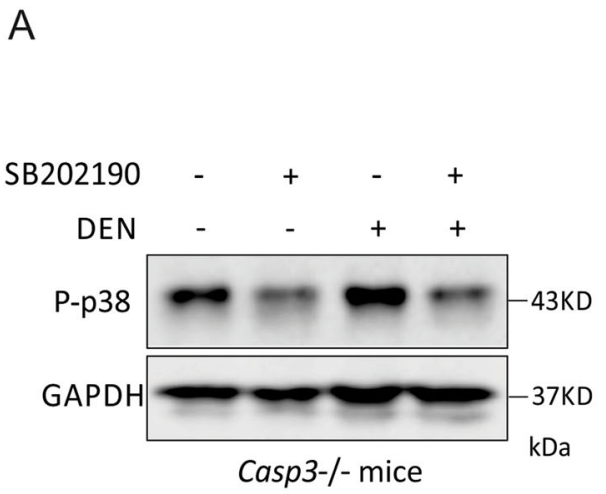

C

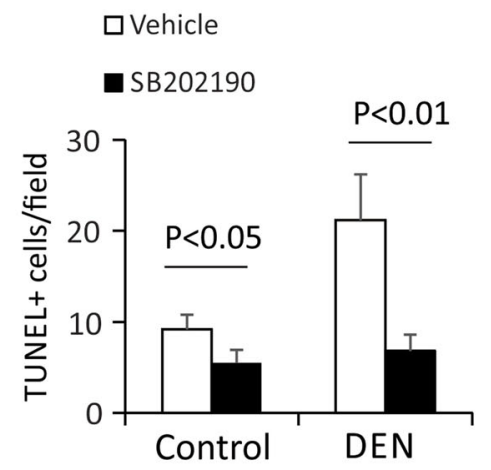

E

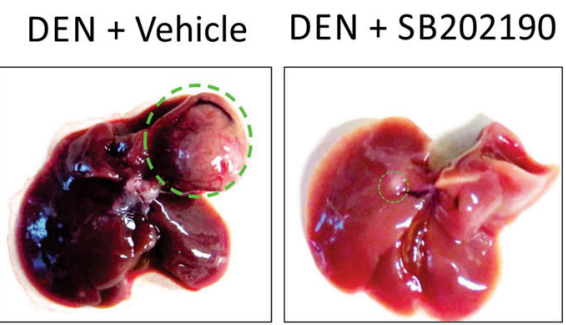

Casp3-/-

\section{B Casp3-/-}
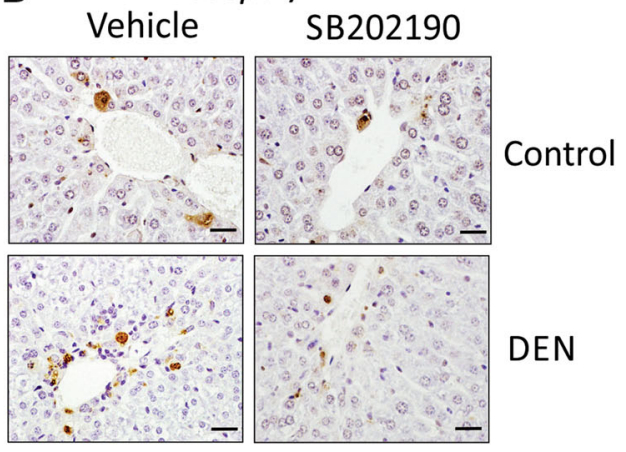

DEN

D $\square$ Vehicle

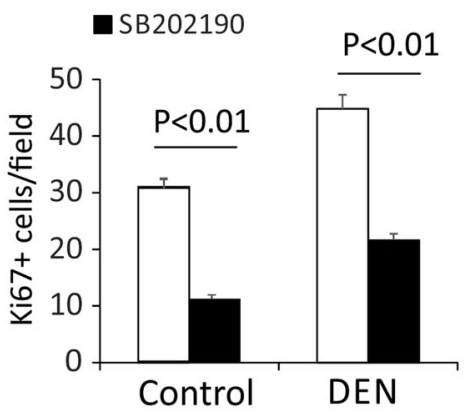

$\mathrm{F}$
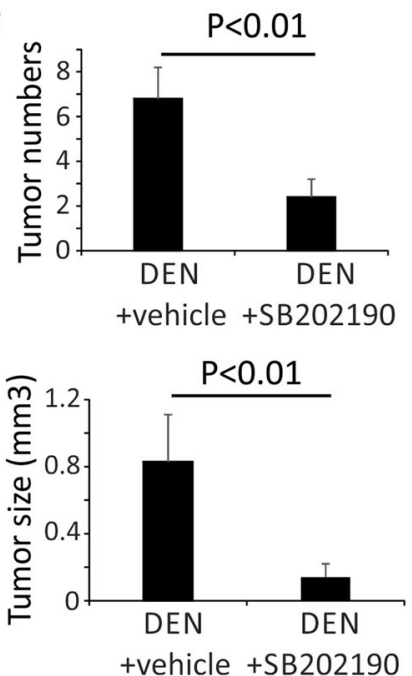

Fig. 5 Inhibition of p38 abrogated enhanced DEN-induced hepatocyte death, compensatory proliferation and HCC development induced by deletion of Caspase-3. a Expression of p-p38 and GAPDH proteins in the livers of Caspase-3 KO mice 3 days following injection with either saline (Un) or $100 \mathrm{mg} / \mathrm{kg}$ DEN plus $20 \mathrm{mg} / \mathrm{kg}$ SB202190 was analyzed by western blotting. b Apoptosis in the livers of Caspase-3 KO mice 3 days following injection with either saline (Un) or $100 \mathrm{mg} / \mathrm{kg}$ DEN plus $20 \mathrm{mg} / \mathrm{kg}$ SB202190 was analyzed by TUNEL staining. Bars: $20 \mu \mathrm{M}$. c Quantification of TUNEL staining for $(\mathbf{b})(n=3)$. d Quantification of Ki67 staining in the livers of Caspase-3 KO mice 3 days after injection with either saline (Un) or $100 \mathrm{mg} / \mathrm{kg}$ DEN plus $20 \mathrm{mg} / \mathrm{kg}$ SB202190. e Photographs of livers of WT and Caspase-3 KO mice 9 months after DEN injection with or without SB202190. $\mathbf{f}$ Quantification of liver tumor numbers $(n=5)$ and liver tumor sizes $(n=5)$ for $(\mathbf{e})$. Values in $(\mathbf{c}),(\mathbf{d})$ and $(\mathbf{f})$ are means \pm SDs

possible that the loss of Caspase-3 increases the activation of MKK6/3 and p38 through regulating its downstream targets. Given the cell context dependence and multiple known targets of Caspase-3, it is not our contention that the increased activation of MKK6/p38 is the only mechanism to explain Caspase-3's effects. Rather, 


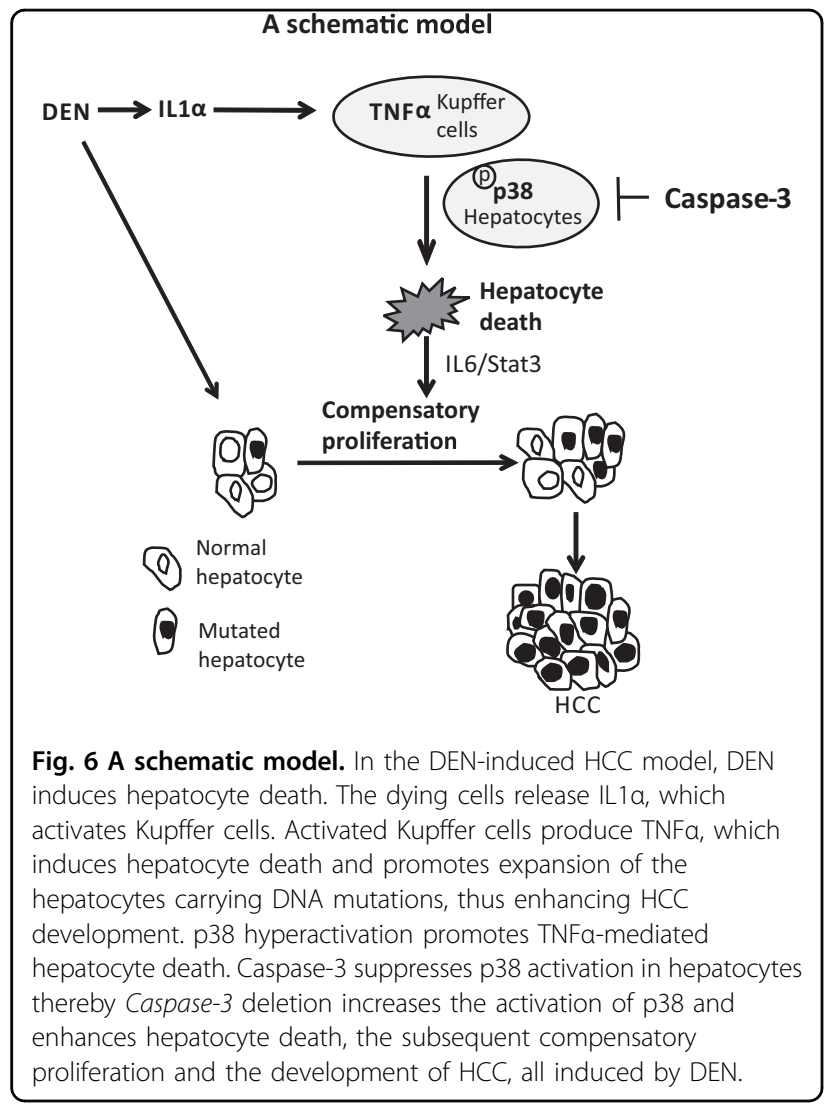

we posit that this mechanism is a contributor to increased hepatocyte death, compensatory proliferation and hepatic carcinogenesis caused by the loss of Caspase3 , and can account for some, if not all, of the functional changes observed.

In conclusion, our study shows that deficiency of Caspase-3 promotes chemical-induced hepatocyte compensatory proliferation and hepatocarcinogenesis. Recent studies suggest that specific inhibition of Casapse-3 in concert with chemotherapy may be a novel approach for the treatment of cancers because of the role of cell death in promoting tissue regeneration in mammalian cells ${ }^{36,37}$. Our data suggest that the role of Caspase- 3 in cancer development is complex and the inhibition of Caspase- 3 might not be suitable for the treatment of HCC patients.

\section{Materials and methods Mice and treatments}

All animals received humane care according to the "Guide for the Care and Use of Laboratory Animals" (http://oacu. od.nih.gov/ac_cbt/guide3.htm). The procedures for all animal experiments were approved by the Institutional Animal Care and Use Committee of Loyola University Chicago. $\mathrm{Casp}^{+/+}$and $\mathrm{Casp3}^{-/-}$littermate mice in a C57BL/6 background were generated from heterozygote intercrosses. Genotyping was performed as described in the JAX Lab website (https://www.jax.org/strain/006233). The mice were housed in micro-isolator cages in a room illuminated from 07:00 a.m. to 07:00 p.m. (12:12-h light-dark cycle), and allowed access to water and chow ad libitum.

For the DEN-induced HCC model, DEN ( $15 \mathrm{mg} / \mathrm{kg})$ was injected intraperitoneally (i.p.) into 15-day-old mice. Mice were sacrificed after 9 months on the standard diet. Surface tumor nodules in each liver lobe were counted and measured with a caliper. For short-term studies of DENinduced liver injury, 8- to 12-week-old mice were injected with DEN (100 mg/kg body weight) i.p. and sacrificed after 3 or 10 days.

For p38 inhibitor treatments, SB202190 (LC Laboratories, Cat \# S-1700) was diluted in 6\% captisol (CyDex, Inc., Lenexa, KS). One day prior to DEN treatment, six 2-week-old or 8- to 12-week-old WT and six 2-week-old or 8- to 12-week-old Casp3 ${ }^{-1-}$ mice were administered vehicle solution (6\% captisol) or $20 \mathrm{mg} / \mathrm{kg} \mathrm{SB202190} \mathrm{by} \mathrm{i.}$ p. daily for 3 days and livers were collected 9 months, 3 or 10 days after DEN treatment.

\section{Isolation and culture of primary mouse hepatocytes}

Hepatocytes from $\mathrm{Casp3}^{+/+}$and $\mathrm{Casp3}^{-/-}$mice were isolated by the non-recirculating two-step perfusion method as previously described ${ }^{38}$. The hepatocytes were then cultured in Williams' medium E supplemented with Hepatocyte Maintenance Supplement Pack (Life Technologies, Grand Island, NY) overnight. Cells were treated with $5 \mathrm{ng} / \mathrm{ml}$ and $20 \mathrm{ng} / \mathrm{ml} \mathrm{IL1} \alpha$ or $5 \mathrm{ng} / \mathrm{ml}$ and $15 \mathrm{ng} / \mathrm{ml}$ TNF $\alpha$ for $1 \mathrm{~h}$; proteins were subsequently collected for western blot analysis.

\section{Culture and treatment of AML12 cells}

AML12 cells were purchased from ATCC and cultured as per the manufacturer's recommendations. Cells were transfected with pCNA3 or pCNA3-p38 $\alpha$ (Addgene, \#20352) plasmids followed by treatment with either saline (vehicle) or $20 \mathrm{ng} / \mathrm{ml}$ TNF $\alpha$ for $48 \mathrm{~h}$. Viability of the cells was analyzed by Alamar Blue Assay.

\section{ALT measurement}

Venous blood of mice was collected from the tail vein 0 , 24 , and $72 \mathrm{~h}$ after DEN treatment. Blood was kept at $4{ }^{\circ} \mathrm{C}$ for overnight and centrifuged at $200 \times g$ for $20 \mathrm{~min}$ to isolate serum. ALT was measured using the Infinity ${ }^{\mathrm{Tm}}$ ALT kit (Thermo Scientific, Middletown, VA) and is reported as mean \pm SD. Briefly, $10 \mu \mathrm{l}$ of serum was added to $100 \mu \mathrm{l}$ ALT reagent and measured at $37^{\circ} \mathrm{C}$ at $340 \mathrm{~nm}$. Each sample was measured in triplicate and three mice were used in each group.

\section{Western blotting}

Western blotting was performed as previously described $^{38}$. Primary antibodies, against Caspase-3 (9662), active 
Caspase-3 (9661), Caspase-6 (9762), active Caspase-6 (9761), Caspase-7 (9492), active Caspase-7 (8438), JNK (9252), p-JNK (9255), NF-kB (8242), p-NF-kB (3033), p38 (8690), p-p38 (4631), p-MK2 (3007), MKK3 (8535), MKK6 (8550), p-MKK3/6 (12280), were purchased from Cell Signaling Technologies (Danvers, MA).

\section{TUNEL staining}

TUNEL staining was performed as previously described $^{38,39}$. The apoptotic index was scored in at least five fields at $\times 400$ magnification/mouse and reported as mean \pm SD. Three mice were used for each group.

\section{IHC staining}

IHC was performed as previously described ${ }^{38,40}$. Cells with positive staining were scored in at least five fields at $\times 400$ or $\times 200$ magnification and reported as mean \pm SD. Three mice were used per group.

\section{Total RNA extraction and real-time reverse transcriptase polymerase chain reaction}

Approximately $100 \mathrm{mg}$ of fresh tissue was minced and put into $600 \mu \mathrm{l}$ lysis buffer (Promega). Total RNA was isolated, and complementary DNA was then generated for real-time PCR analysis as described ${ }^{40}$. Real-time PCR was performed on a Mini Opticon Real-time PCR system (BioRad) with SYBR Green (Invitrogen) and specific primers ${ }^{19}$. Melting curves and agarose gel electrophoresis of the PCR products were used to verify the specificity of PCR amplification.

\section{Statistical analysis}

Statistical analysis was performed using GraphPad Prism V software. Data are presented as means \pm standard deviations (SD). Statistical significance was calculated using the Student's $t$-test. $P<0.05$ was considered to be significant. The means \pm SDs are shown in figures where applicable.

\section{Acknowledgements}

We thank Drs. Mitchell F. Denning, Nancy Zeleznik-Le, Jiwang Zhang and Manuel Diaz (all of Loyola University Chicago) for their helpful discussions and advice. This work is supported in part by AASLD Liver Scholar Award (W.Q.), NIH R03CA195183 (W.Q.), R03CA184652 (W.Q.) and R01CA197128 (W.Q.).

\footnotetext{
Author details

${ }^{1}$ Department of Surgery and Oncology Institute, Loyola University Chicago Stritch School of Medicine, 2160 South 1st Avenue, Maywood, IL 60153, USA. ${ }^{2}$ Department of Pathology, Loyola University Chicago Stritch School of Medicine, 2160 South 1st Avenue, Maywood, IL 60153, USA. ${ }^{3}$ Department of Molecular/Cellular Physiology, Loyola University Chicago Stritch School of Medicine, 2160 South 1st Avenue, Maywood, IL 60153, USA. ${ }^{4}$ Department of Applied and Computational Mathematics and Statistics, University of Notre Dame, Notre Dame, IN, USA. ${ }^{5}$ Department of General Surgery Tongji Hospital, Tongji University Medical School, Shanghai, PR 200065, China
}

\section{Authors' contributions}

N.S. performed experiments, analyzed data and wrote the paper. T.B. bred mice and performed experiments. P.B. wrote the paper. X.Z.D. analyzed histology. J.L. performed statistical analysis. B.M.S. designed experiments, analyzed data and wrote the paper. W.Q. designed and performed experiments, analyzed data and wrote the paper.

\section{Conflict of interest}

The authors declare that they have no conflict of interest.

\section{Publisher's note}

Springer Nature remains neutral with regard to jurisdictional claims in published maps and institutional affiliations.

Supplementary Information accompanies this paper at (https://doi.org/ 10.1038/s41419-018-0617-7)

Received: 12 April 2018 Accepted: 19 April 2018

Published online: 11 May 2018

\section{References}

1. Bruix, J. \& Sherman, M., Practice Guidelines Committee, A.A.f.t.S.o.L.D. Management of hepatocellular carcinoma. Hepatology 42, 1208-1236 (2005).

2. Johnson, P. J. Non-surgical treatment of hepatocellular carcinoma. HPB 7, 50-55 (2005).

3. Llovet, J. M. et al. Sorafenib in advanced hepatocellular carcinoma. N. Engl. J. Med. 359, 378-390 (2008).

4. Bruix, J., Boix, L., Sala, M. \& Llovet, J. M. Focus on hepatocellular carcinoma. Cancer Cell. 5, 215-219 (2004).

5. Maeda, S., Kamata, H., Luo, J. L., Leffert, H. \& Karin, M. IKKbeta couples hepatocyte death to cytokine-driven compensatory proliferation that promotes chemical hepatocarcinogenesis. Cell 121, 977-990 (2005).

6. He, G. \& Karin, M. NF-kappaB and STAT3 - key players in liver inflammation and cancer. Cell Res. 21, 159-168 (2011).

7. Schulte-Hermann, R., Bursch, W., Low-Baselli, A., Wagner, A. \& Grasl-Kraupp, B. Apoptosis in the liver and its role in hepatocarcinogenesis. Cell. Biol. Toxicol. 13, 339-348 (1997)

8. Farber, E. Hepatocyte proliferation in stepwise development of experimental liver cell cancer. Dig. Dis. Sci. 36, 973-978 (1991).

9. Aravalli, R. N., Cressman, E. N. \& Steer, C. J. Cellular and molecular mechanisms of hepatocellular carcinoma: an update. Arch. Toxicol. 87, 227-247 (2012).

10. Weber, A., Boege, Y., Reisinger, F. \& Heikenwalder, M. Chronic liver inflammation and hepatocellular carcinoma: persistence matters. Swiss Med. Wkly. 141, w13197 (2011)

11. Sakurai, T., Maeda, S., Chang, L. \& Karin, M. Loss of hepatic NF-kappa B activity enhances chemical hepatocarcinogenesis through sustained c-Jun N-terminal kinase 1 activation. Proc. Natl. Acad. Sci. USA 103, 10544-10551 (2006).

12. Schwabe, R. F. et al. c-Jun-N-terminal kinase drives cyclin D1 expression and proliferation during liver regeneration. Hepatology 37, 824-832 (2003).

13. Hui, L., Zatloukal, K., Scheuch, H., Stepniak, E. \& Wagner, E. F. Proliferation of human HCC cells and chemically induced mouse liver cancers requires JNK1dependent p21 downregulation. J. Clin. Invest. 118, 3943-3953 (2008).

14. Seki, E., Brenner, D. A. \& Karin, M. A liver full of JNK: signaling in regulation of cell function and disease pathogenesis, and clinical approaches. Gastroenterology 143, 307-320 (2012).

15. Sakurai, T. et al. Hepatocyte necrosis induced by oxidative stress and IL-1 alpha release mediate carcinogen-induced compensatory proliferation and liver tumorigenesis. Cancer Cell. 14, 156-165 (2008).

16. Naugler, W. E. et al. Gender disparity in liver cancer due to sex differences in MyD88-dependent IL-6 production. Science 317, 121-124 (2007).

17. Schwabe, R. F. \& Brenner, D. A. Mechanisms of Liver Injury. I. TNF-alphainduced liver injury: role of IKK, JNK, and ROS pathways. Am. J. Physiol. Gastrointest. Liver. Physiol. 290, G583-G589 (2006).

18. Johnson, C. et al. Interleukin-6 and its receptor, key players in hepatobiliary inflammation and cancer. Transl. Gastrointest. Cancer 1, 58-70 (2012).

19. Qiu, W. et al. PUMA-mediated apoptosis drives chemical hepatocarcinogenesis in mice. Hepatology 54, 1249-1258 (2011). 
20. Crawford, E. D. \& Wells, J. A. Caspase substrates and cellular remodeling. Annu. Rev. Biochem. 80, 1055-1087 (2011)

21. Parrish, A. B., Freel, C. D. \& Kornbluth, S. Cellular mechanisms controlling caspase activation and function. Cold Spring Harb. Perspect. Biol. 5, 1-25 (2013)

22. Feinberg, A. \& Zedeck, M. S. Production of a highly reactive alkylating agent from the organospecific carcinogen methylazoxymethanol by alcohol dehydrogenase. Cancer Res. 40, 4446-4450 (1980).

23. Shi, Y. Caspase activation, inhibition, and reactivation: a mechanistic view. Protein Sci. 13, 1979-1987 (2004).

24. Karin, M. NF-kappaB as a critical link between inflammation and cancer. Cold Spring Harb. Perspect. Biol. 1, a000141 (2009).

25. Breitwieser, W. et al. Feedback regulation of p38 activity via ATF2 is essential for survival of embryonic liver cells. Genes Dev. 21, 2069-2082 (2007).

26. Chen, Z. J., Parent, L. \& Maniatis, T. Site-specific phosphorylation of IkappaBalpha by a novel ubiquitination-dependent protein kinase activity. Cell $\mathbf{8 4}$ 853-862 (1996).

27. Hein, $H$. et al. Genomic organization, sequence, and transcriptional regulation of the human eotaxin gene. Biochem. Biophys. Res. Commun. 237, 537-542 (1997).

28. Pahl, H. L. Activators and target genes of Rel/NF-kappaB transcription factors. Oncogene 18, 6853-6866 (1999).

29. Koul, H. K. Pal, M. \& Koul, S. Role of p38 MAP kinase signal transduction in solid tumors. Genes Cancer 4, 342-359 (2013).

30. lyoda, K. et al. Involvement of the p38 mitogen-activated protein kinase cascade in hepatocellular carcinoma. Cancer 97, 3017-3026 (2003).
31. Kang, J. S. et al. DBM1285 suppresses tumor necrosis factor alpha production by blocking p38 mitogen-activated protein kinase/mitogen-activated protein kinase-activated protein kinase 2 signaling pathway. J. Pharmacol. Exp. Ther. 334, 657-664 (2010).

32. Huang, $\mathrm{H}$. et al. Expression and prognostic significance of osteopontin and caspase-3 in hepatocellular carcinoma patients after curative resection. Cancer Sci. 101, 1314-1319 (2010)

33. Mcllwain, D. R., Berger, T. \& Mak, T. W. Caspase functions in cell death and disease. Cold Spring Harb. Perspect. Biol. 5, a008656 (2013).

34. Enslen, H., Raingeaud, J. \& Davis, R. J. Selective activation of p38 mitogenactivated protein (MAP) kinase isoforms by the MAP kinase kinases MKK3 and MKK6. J. Biol. Chem. 273, 1741-1748 (1998).

35. Fischer, U., Janicke, R. U. \& Schulze-Osthoff, K. Many cuts to ruin: a comprehensive update of caspase substrates. Cell Death Differ. 10, 76-100 (2003).

36. Li, F. et al. Apoptotic cells activate the "phoenix rising" pathway to promote wound healing and tissue regeneration. Sci. Signal. 3, ra13 (2010).

37. Boland, K., Flanagan, L. \& Prehn, J. H. Paracrine control of tissue regeneration and cell proliferation by Caspase-3. Cell Death Dis. 4, e725 (2013).

38. Shang, N. et al. FAK is required for c-Met/beta-catenin-driven hepatocarcinogenesis. Hepatology 61, 214-226 (2015).

39. Shang, N. et al. FAK kinase activity is required for the progression of c-Met/ beta-catenin-driven HCC. Gene Exp. 17, 79-88 (2016).

40. Arteaga, M. et al. Inhibition of SIRT2 suppresses hepatic fibrosis. Am. J. Physiol. Gastrointest. Liver. Physiol. 310, G1155-G1168 (2016). 